\title{
ZEITSCHRIFT \\ DER SAVIGNY-STIFTUNG \\ FÜR \\ RECHTSGESCHICHTE
}

\author{
128. B A N D
}

\begin{abstract}
H E R A U S G E E B E N VON
W. KAISER, M. SCHERMAIER, G. THÜR, G. KÖBLER, P. OESTMANN, J. RÜCKERT, H.-J. BECKER, H. DE WALL, A. THIER
\end{abstract}

KANONISTISCHE ABTEILUNG 97

DIE ZRG ERSCHEINT SEIT I5 O JAHREN, DIE KA SEIT IOO JAHREN.

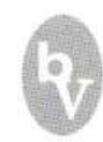

2011

B ÖHLAU VERLAG WIEN KÖLN WEIMAR 
Die Zeitschrift der Savigny-Stiftung für Rechtsgeschichte erscheint jährlich in drei selbständigen, auch einzeln käuflichen Abteilungen. Sie veröffentlicht Beiträge zur rechtshistorischen Forschung und berichtet über das einschlägige wissenschaftliche Schrifttum.

Richtlinien für die Manuskriptgestaltung finden Sie in diesem Band der ZRG S. 588, sowie auf www.boehlau-verlag.com. Redaktion der ZRG: DDr. Reingard Rauch, Waldheimatweg 33, A-8010 Graz, reingard.rauch@boehlau.at

Die Herausgeber und ihre Anschriften:

Romanistische Abteilung: Prof. Dr. Gerhard Thür, Kommission für Antike Rechtsgeschichte, Postgasse 7-9, A-1010 Wien, gerhard.thuer@oeaw.ac.at (Aufsätze und Miszellen aus dem Bereich der Antiken Rechtsgeschichte); Prof. Dr. Martin J. Schermaier, Institut für Römisches Recht und Vergleichende Rechtsgeschichte, Rheinische Friedrich-WilhelmsUniversität, Adenauerallee 24-42, D-53113 Bonn, schermaier@jura.uni-bonn.de (Aufsätze und Miszellen aus dem Bereich des Römischen Rechts und der Privatrechtsgeschichte) und Prof.Dr. Wolfgang Kaiser, Institut für Rechtsgeschichte und geschichtliche Rechtsvergleichung - Romanistische Abteilung, Albert-Ludwigs-Universität, Platz der Alten Synagoge, D-79085 Freiburg, wolfgang.kaiser@jura.uni-freiburg.de (Besprechungen)

Germanistische Abteilung: Prof. Dr. Peter Oestmann, Institut für Rechtsgeschichte, Westfälische Wilhelms-Universität, Universitätsstraße 14-16, D-48143 Münster, oestmann@ uni-muenster.de (Aufsätze und Miszellen für die Zeit vor 1800); Prof. Dr. Joachim Rückert, Lehrstuhl für Neuere Rechtsgeschichte, Juristische Zeitgeschichte, Zivilrecht und Rechtsphilosophie, Goethe-Universität FB 01 Fach 13, Postfach 1119 32, D-60054 Frankfurt, rueckert@jur.uni-frankfurt.de (Aufsätze und Miszellen zur Rechtsgeschichte des 19. und 20. Jahrhunderts); Prof. Dr. Gerhard Köbler, Universität, Innrain 52, A-6020 Innsbruck, gerhard.koebler@uibk.ac.at (Besprechungen bis 15.11.2011); Prof. Dr. Hans-Peter Haferkamp, Direktor Inst. f. neuere Privatrechtsgeschichte, Deutsche und Rheinische Rechtsgeschichte, Universität zu Köln, Albertus-Magnus-Platz, D-50923 Köln, hans-peter.haferkamp @uni-koeln.de (Besprechungen ab 15.11.2011)

Kanonistische Abteilung: Prof. Dr. Andreas Thier M. A., Rechtswissenschaftliches Institut, Universität Zürich, Rämistraße 74, CH-8001 Zürich, Ist.thier@rwi.uzh.ch (Kanonisches Recht bis ca. 1400); Prof. Dr. Hans-Jürgen Becker, Lehrstuhl für Bürgerliches Recht, Europäische Rechtsgeschichte und Kirchenrecht, Universitätsstraße 31, D-93040 Regensburg, hans-juergen.becker@jura.uni-regensburg.de (Kanonisches Recht nach 1400); Prof. Dr. Heinrich de Wall, Hans-Liermann-Institut, Hindenburgstraße 34, D-91054 Erlangen, hli@ jura.uni-erlangen.de (Evangelisches Kirchenrecht und Staatskirchenrecht)

Gedruckt mit freundlicher Unterstützung des Bundesministeriums für Wissenschaft und Forschung, Wien

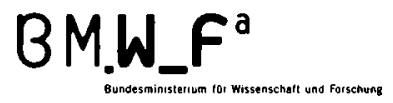

ISSN 0323-4142

ISBN 978-3-205-78774-7 (Einzelband)

(C) 2011 Böhlau Verlag, Dr. Peter Rauch G.m.b.H., A-1010 Wien. Alle Rechte vorbehalten.

Satz: Vogelmedia $\mathrm{GmbH}, \mathrm{A}-2102$ Bisamberg

Druck und Herstellung: Prime Rate kft., Budapest 